\title{
Parâmetros de metabólitos bioquímicos em ovinos criados no Brasil
}

\author{
Débora Adriana de Paula Silva ${ }^{1}$; Laura Ferrari Monteiro Varanis ${ }^{2}$; Karla Alves Oliveira ${ }^{3 *}$; Luciana Melo Sousa \\ 4; Marco Tulio Santos Siqueira ${ }^{5}$; Gilberto de Lima Macedo Júnior ${ }^{6}$
}

DOI: https://doi.org/10.35699/2447-6218.2020.20404

\begin{abstract}
Resumo
O trabalho teve como objetivo definir intervalos de referência para valores de metabólitos energéticos, proteicos e enzimáticos em ovinos. Os dados utilizados foram oriundos de vários experimentos realizados entre 2006 e 2018, em diversas instituições de ensino superior e pesquisa. Foi utilizado banco de dados diversificado com observações de ovinos de diferentes raças, genótipos, idades e categorias fisiológicas, criados em diferentes sistemas de manejo. Todos os animais utilizados eram saudáveis e dados de animais que apresentaram quaisquer manifestações clínicas foram descartados. Para determinar o perfil metabólico energético foram obtidos dados de glicose, colesterol, triglicerídeos, frutosamina, HDL (lipoproteína de alta densidade), LDL (lipoproteína de baixa densidade) e VLDL (lipoproteína de muito baixa densidade); para o perfil proteico, dados de proteínas totais, ácido úrico, ureia, albumina e creatinina; e para o enzimático, dados de AST (aspartato aminotransferase), GGT (gama glutamil transferase) e FA (fosfatase alcalina). As análises laboratoriais foram realizadas nos aparelhos Analisador bioquímico semiautomático modelo BIO - 2000 da marca Bioplus ${ }^{\circledast}$ e Analisador automático de bioquímica modelo PKL-125 da marca MHLab ${ }^{\circledR}$. Para a estimativa e determinação dos valores de referência, foi utilizado programa RefVal 4.11, sendo os valores outliers removidos da avaliação. Após a determinação dos valores de referência comparou-se os dados obtidos com tabelas internacionais estabelecidas na literatura, sendo possível observar que os intervalos encontrados neste trabalho foram mais amplos, abrangendo melhor resultados obtidos em experimentos realizados no Brasil.
\end{abstract}

Palavras-chave: Ovis aries. Parâmetros sanguíneos. Perfil energético. Perfil proteico.

\section{Biochemical Metabolite Parameters for Sheep in Brazil}

\begin{abstract}
The objective was to define reference intervals for values of energetic, protein, and enzymatic metabolites in sheep. The data used came from several experiments carried out between 2006 and 2018, in several higher education and research institutions. A diversified database was used with observations of sheep of different breeds, genotypes, ages and physiological categories, created in different management systems. All animals used were healthy and data from animals that showed any clinical manifestations were discarded. In order to determine the metabolic energy profile, glucose, cholesterol, triglycerides, fructosamine, HDL (high density lipoprotein), LDL (low density lipoprotein) and VLDL (very low density lipoprotein) data were obtained; for the protein profile, data on total proteins, uric acid, urea,
\end{abstract}

${ }^{1}$ Universidade Federal de Uberlândia. Uberlândia, MG. Brasil.

https://orcid.org/0000-0003-3052-0544

${ }^{2}$ Universidade Federal de Uberlândia. Uberlândia, MG. Brasil.

https://orcid.org/0000-0003-1562-4505

${ }^{3}$ Universidade Estadual Paulista - UNESP campus Jaboticabal. Jaboticabal, SP. Brasil.

https://orcid.org/0000-0002-7792-2615

${ }^{4}$ Universidade Federal de Uberlândia. Uberlândia, MG. Brasil. https://orcid.org/0000-0003-1016-8248

${ }^{5}$ Universidade Federal de Uberlândia. Uberlândia, MG. Brasil. https://orcid.org/0000-0002-2098-8568

${ }^{6}$ Universidade Federal de Uberlândia. Uberlandia, MG. Brasil.

https://orcid.org/0000-0001-5781-7917

*Autor para correspondência: karla.alves.oliveira@hotmail.com

Recebido para publicação em 01 de Maio de 2020. Aceito para publicação 10 de Setembro de 2020 e-ISSN: 2447-6218 / ISSN: 2447-6218. Atribuição CC BY. 
Silva, D. A. P. et al.

albumin and creatinine; and for the enzymatic profile, AST (aspartate aminotransferase), GGT (gamma glutamyl transferase) and AP (alkaline phosphatase) data. Laboratory analyzes were carried out on the Bioplus ${ }^{\odot}$ semiautomatic biochemical analyzer model, Bioplus ${ }^{\odot}$, and automatic biochemistry analyzer, model PKL-125, MHLab ${ }^{\circledR}$. For the estimation and determination of the reference values, the RefVal 4.11 program was used, and the outliers values were removed from the evaluation. After determining the reference values, the data obtained were compared with international tables established in the literature, and it is possible to observe that the intervals found in this work were broader, covering better results obtained in experiments carried out in Brazil.

Keywords: Blood parameters. Energetic profile. Ovis aries. Proteic profile.

\section{Introdução}

Na década de 1970, Payne et al. (1970) elaboraram o termo perfil metabólico, isto é, a análise de componentes hemato-bioquímicos do plasma sanguíneo aplicada a populações, a fim de diagnosticar, avaliar e prevenir transtornos metabólicos, além de indicar o status nutricional do rebanho.

Apesar de uma ferramenta muito útil, uma das maiores dificuldades da aplicação correta do perfil metabólico é a interpretação dos dados, que se torna complicada, tanto pela complexidade dos mecanismos fisiológicos que regulam os níveis sanguíneos dos metabólitos que também podem sofrer influência de fatores ambientais quanto pela falta de valores de referência que se adequem à situação analisada. Para que seja feita uma interpretação correta, é necessário conhecer os valores de referência adequados para cada região, espécie e população em questão (Varanis, 2018; Libardi et al., 2018)

Há na literatura uma variedade de trabalhos internacionais e também alguns nacionais estimando valores de referência para ovinos, sendo um exemplo o de Varanis (2018) que preconizou intervalos de referência para metabólitos energéticos, proteicos, enzimáticos e minerais para ovinos em diferentes categorias fisiológicas. Entretanto, dentre os vários intervalos de referência encontrados na literatura, os mais comumente adotados ainda são internacionais, como os preconizados por Kaneko et al., (2008) no livro Clinical Biochemistry of Domestic Animals, um dos mais citados na literatura com cerca de 5.000 citações (Google Scholar, 2019)

Os rebanhos brasileiros possuem diversas particularidades, desde a nutrição, onde é comum a utilização de produtos e subprodutos de ingredientes encontrados apenas em determinadas regiões, a utilização de raças nacionais frutos de cruzamentos não especificados, diferenças de manejo e ambientação, onde os animais se adaptam a clima, temperatura, pluviosidade, características de solo e instalações únicas para cada região. Logo, a avaliação desses rebanhos com base em tabelas internacionais, obtidas a partir de dados de animais criados muitas vezes em sistemas que nem seriam possíveis de se aplicar no Brasil pode não ser adequada, podendo ocasionar diagnósticos e avaliações equivocadas. Isso evidencia uma lacuna de informações de origem nacional que possam ser empre- gadas na avaliação metabólica de ovinos, possibilitando a proposição de uma tabela de valores de referência que melhor reflita a atual condição dos animais em nosso país, sendo confeccionada com dados obtidos nas diferentes regiões do país abrangendo as variações encontradas na ovinocultura nacional, e também respeitando as normas propostas para a determinação de tais valores.

Diante disso, o objetivo do trabalho foi determinar intervalos de referência para os valores de metabólitos energéticos, proteicos, minerais e enzimáticos para ovinos e compará-los com os valores de referência obtidos na literatura internacional.

\section{Material e métodos}

Para a estimativa dos valores de referência compôs-se um banco de dados bastante diversificado, com observações obtidas de experimentos conduzidos em diversas instituições, fazendas comerciais, sistemas de manejo, genótipos e categorias fisiológicas. Foram coletados dados da Universidade Federal de Uberlândia, Universidade Federal de Minas Gerais, Universidade Federal de Lavras e Universidade Federal Rural do Rio de Janeiro. Na região norte foram obtidos dados da Universidade Federal do Tocantins e de duas propriedades comerciais. Essa diversificação nos dados obtidos permite observar com maior acurácia a amplitude nos valores metabólicos. Até porque usamos dados internacionais onde não são descritos como foram obtidos em grande parte da literatura. Os experimentos foram conduzidos no período de 2006 à 2018 com ovinos criados em diferentes sistemas de manejo: a pasto, confinamento total, semi confinamento, confinamento coletivo e/ou individual. Esses manejos são utilizados em criações por todo o Brasil. Os dados aqui apresentados retratam com amplitude os diferentes tipos de manejos (alimentar, nutricional e manejo geral). Todos os dados apresentados nesse trabalho tiveram as devidas autorizações de seus autores. O número amostral está nas tabelas que apresentam os valores referenciais obtidos.

Para a realização destes experimentos foram utilizados animais de diferentes raças e genótipos, como Lacaune, Santa Inês, Dorper, White Dorper, Morada Nova, Texel, Somalis e também cruzamentos entre essas raças. 
Parâmetros de metabólitos bioquímicos em ovinos criados no Brasil

Utilizaram-se também machos e fêmeas de diversas categorias fisiológicas: cordeiros, borregos, machos castrados e inteiros, fêmeas vazias, prenhas e lactantes. Todos os animais eram saudáveis, não passaram por condições de desnutrição forçada e dados de animais que apresentaram quaisquer manifestações clínicas foram descartados. Os dados foram coletados, agrupados, e um banco de dados foi confeccionado para cada metabólito.

Para determinar o perfil metabólico energético, foram obtidos dados de glicose, colesterol, triglicerídeos, frutosamina, HDL (lipoproteína de alta densidade), LDL (lipoproteína de baixa densidade) e VLDL (lipoproteína de muito baixa densidade). Os valores de VLDL e LDL foram obtidos por cálculos propostos por Friedewald, Lew e Fredrickson (1972) a partir dos valores de colesterol total, HDL-colesterol e triglicerídeos, conforme as equações (Eq. 1) e (Eq. 2):

$$
\begin{gathered}
V L D L=T G / 5 \\
L D L=C T-H D L-V L D L
\end{gathered}
$$

Onde: $\mathrm{VLDL}=$ lipoproteína de muito baixa densidade; $\mathrm{TG}=$ triglicerídeos; $\mathrm{LDL}=$ lipoproteína de baixa densidade; $\mathrm{CT}=$ colesterol total; $\mathrm{HDL}=$ lipoproteína de alta densidade.
Para o perfil proteico foram utilizados dados de proteínas totais, ácido úrico, ureia, albumina e creatinina; e para o perfil enzimático, dados de AST (aspartato aminotransferase), GGT (gama glutamil transferase), fosfatase alcalina. As análises laboratoriais foram realizadas nos aparelhos Analisador bioquímico semiautomático modelo BIO - 2000 da marca Bioplus ${ }^{\odot}$ e Analisador automático de bioquímica modelo PKL-125 da marca MHLab ${ }^{\circledR}$, utilizando kits de diferentes marcas (Labtest Diagnóstica S. A., Biotecnica ${ }^{\odot}$, GT Group ${ }^{\odot}$ ).

Para a estimativa e determinação dos valores de referência, foi utilizado programa RefVal 4.11 (Solberg, 2006). Os valores outliers foram removidos, utilizando o teste de Dixon, e os percentis, assim como seus intervalos de confiança, estimados pelo método de boostraping não paramétrico, quando os dados não apresentaram distribuição normal. O intervalo de confiança definido foi de $95 \%$.

\section{Resultados e discussão}

\section{Perfil energético}

Para estabelecer o perfil energético, coletaram-se dados de glicose, colesterol, triglicerídeos, e frutosamina, HDL, LDL e VLDL (Tabela 1).

\begin{tabular}{|c|c|c|c|c|}
\hline Metabólito & $\mathrm{N}^{1}$ & $\begin{array}{c}\text { Intervalos definidos nes- } \\
\text { te estudo }\end{array}$ & $\begin{array}{l}\text { Intervalos definidos por Ka- } \\
\text { neko et al., (2008) }\end{array}$ & Unidade \\
\hline Glicose & 3005 & $30-94$ & $50-80$ & $\mathrm{mg} \mathrm{dL}{ }^{-1}$ \\
\hline Colesterol & 4486 & $14-126$ & $52-76$ & $\mathrm{mg} \mathrm{dL} \mathrm{L}^{-1}$ \\
\hline Triglicerídeos & 3936 & $5-71$ & $9-30$ & $\mathrm{mg} \mathrm{dL} \mathrm{L}^{-1}$ \\
\hline Frutosamina & 1883 & $119-451$ & $170-174$ & $\mu \mathrm{mol} \mathrm{L}^{-1}$ \\
\hline $\mathrm{HDL}^{1}$ & 1656 & $10-76,7$ & $\mathrm{SIL}^{4}$ & $\mathrm{mg} \mathrm{dL} \mathrm{L}^{-1}$ \\
\hline $\mathrm{LDL}^{2}$ & 1242 & $1,2-87$ & $\mathrm{SIL}^{4}$ & $\mathrm{mg} \mathrm{dL} \mathrm{L}^{-1}$ \\
\hline VLDL $^{3}$ & 3424 & $1-16,4$ & SIL $^{4}$ & $\mathrm{mg} \mathrm{dL} \mathrm{L}^{-1}$ \\
\hline
\end{tabular}

Tabela 1 - Intervalos de referência para metabólitos energéticos de ovinos

1 - HDL - Lipoproteína de alta densidade; ${ }^{2}$ - LDL - Lipoproteína de baixa densidade; ${ }^{3}$ - VLDL - Lipoproteína de muito baixa densidade; ${ }^{4}$ SIL - Sem informações na literatura. $\mathrm{N}^{1}$ - número amostral

A amplitude obtida para o intervalo de glicose foi de $64 \mathrm{mg} \mathrm{dL}^{-1}$ enquanto a amplitude do intervalo de Kaneko e al. (2008) é de $30 \mathrm{mg} \mathrm{dL}{ }^{-1}$, sendo menos da metade. Nos ruminantes, a manutenção da concentração de glicose plasmática se relaciona a estabilidade glicêmica, visto que os carboidratos da dieta são quase totalmente utilizados no rúmen (Gressler et al., 2015). Alterações fisiológicas como a prenhez também influenciam na concentração sanguínea de glicose, assim como fatores ambientais como o estresse, que por sua vez é capaz de alterar a dinâmica hormonal que regula a gliconeogênese e a utilização celular da glicose, podendo elevar os níveis na corrente (Kozloski, 2017).
Diversos trabalhos nacionais apresentam resultados condizentes com os intervalos definidos, por exemplo, Oliveira et al., (2014) em estudo com ovelhas Santa Inês gestantes criadas em sistema semi-intensivo, encontrou valores entre 38,58 e $44,29 \mathrm{mg} \mathrm{dL}^{-1} \mathrm{em}$ diferentes fases gestacionais, esses resultados estão abaixo dos valores de referência definidos por Kaneko et al., (2008), entretanto se encontram dentro do intervalo definido neste estudo. Ribeiro et al., (2003) trabalhando com borregas Corriedale de 4 meses de idade em um estudo comparativo entre diferentes estações do ano no Rio Grande do Sul, encontrou uma média de glicose sanguínea de $52,18 \mathrm{mg} \mathrm{dL}^{-1}$. Rabassa et al., (2009) trabalhando com ovelhas Corriedale a pasto encontrou um valor médio de 
Silva, D. A. P. et al.

glicose de 38,91 $\mathrm{mg} \mathrm{dL}^{-1}$. Alvarenga (2011) trabalhando com carneiros adultos de seis espécies diferentes, criados em sistema semi-intensivo no Distrito Federal encontrou valores de glicose variando entre 50,5 e $53,5 \mathrm{mg} \mathrm{dL}^{-1}$.

Para os dados de colesterol obtivemos amplitude de $112 \mathrm{mg} \mathrm{dL}^{-1}$, enquanto o de Kaneko et al., (2008) possui amplitude de $24 \mathrm{mg} \mathrm{dL}^{-1}$, 4,6 vezes menor. De acordo com Silva (2019) os níveis de colesterol plasmático são uma boa ferramenta de avaliação do balanço energético. Nos ruminantes a biossíntese do colesterol acontece principalmente no intestino delgado e no tecido adiposo, a partir do acetil-CoA originado do ácido acético, que é produzido no rúmen através da fermentação da fibra (Kaneko et al., 2008). Logo, sugere-se que a maior amplitude obtida neste trabalho pode estar relacionada a predominância de forragens na dieta dos animais utilizados.

Na literatura encontra-se trabalhos cujos resultados condizem com os intervalos encontrados neste estudo, como o de Paula (2015) que utilizando ovelhas adultas sem padrão racial definido, alimentadas com níveis crescentes de melaço de soja, encontrou valores de colesterol entre 51,72 e 61,0 mg dL ${ }^{-1}$ Rabassa et al., (2009) em estudo com ovelhas adultas da raça Corriedale criadas a pasto no Rio Grande do Sul obtiveram uma média de $37,25 \mathrm{mg} \mathrm{dL}^{-1}$ entre os animais. Balaro, Cardoso e Peneiras (2012) trabalhando com cordeiros Santa Inês a pasto no estado do Rio de Janeiro obtiveram média de $70,05 \mathrm{mg} \mathrm{dL}^{-1}$. Homem Junior et al., (2010) trabalhando também com cordeiros Santa Inês no estado de São Paulo, encontraram médias de colesterol entre 42,9 e 108,6 mg $\mathrm{dL}^{-1}$. Com isso, é possível observar que todos os resultados apresentados estariam fora do intervalo de normalidade sugerido por Kaneko et al., (2008), entretanto se enquadram nos resultados obtidos no presente trabalho.

A partir dos dados de triglicerídeos obtivemos um intervalo de $66 \mathrm{mg} \mathrm{dL}^{-1}$ de amplitude enquanto o intervalo de Kaneko et al., (2008) possui amplitude de $21 \mathrm{mg} \mathrm{dL}^{-1}, 3,14$ vezes menor. Os triglicerídeos são a principal forma de armazenamento de energia no organismo animal. São sintetizados em quase todos os tecidos, se destacando no fígado e no tecido adiposo. A biossíntese dos triglicerídeos é feita a partir da glicose circulante e do glicerol, logo suas concentrações se relacionam com as de glicose (Angeli, 2014). Brito et al., (2006) trabalhando com ovelhas Lacaune no Rio Grande do Sul obtiveram médias de triglicerídeos entre 28,7 e 52,2 $\mathrm{mg} \mathrm{dL}^{-1}$. Sá et al., (2014) em estudo com borregos sem padrão racial definido, confinados no estado do Tocantins, encontraram médias entre 30,51 e 40,28 $\mathrm{mg} \mathrm{dL}^{-1}$. Santos et al., (2015) trabalhando em Minas Gerais com cordeiros Santa Inês de até 90 dias de idade encontrou média de 34,72 mg $\mathrm{dL}^{-1}$. É possível observar que os resultados encontrados advêm de animais de raças e idades distintas, criados em diferentes estados, em condições ambientais condizentes com a localidade dos experimentos, havendo variações entre essas respostas e a maioria sendo acima do limite preconizado por Kaneko et al (2008), porém, todos eles se enquadram no intervalo de normalidade definido neste estudo.

O intervalo definido para o metabólito Frutosamina apresentou maior diferença de amplitude entre os metabólitos energéticos, sendo $332 \mu \mathrm{mol} \mathrm{L}-1$ enquanto a amplitude do intervalo de Kaneko et al., (2008) é de apenas $4 \mu \mathrm{mol} \mathrm{L}{ }^{-1}$. Os níveis de frutosamina sofrem influência tanto do metabolismo energético quanto proteico, visto que ela é formada por uma molécula de glicose conjugada a uma proteína, geralmente a albumina. Em contrapartida à instabilidade da glicose, a albumina tem um turnover de cerca de 30 dias, conferindo maior estabilidade a molécula de frutosamina, ou seja, fazendo com que ela permaneça na corrente por um período de tempo maior. Por depender da concentração sanguínea de glicose, a frutosamina sofre alterações advindas dos mesmos fatores, portanto é possível relacionar a variação do intervalo de frutosamina a variação do intervalo de glicose, considerando o tempo maior de permanência desta na corrente sanguínea (Varanis, 2018; Kaneko et al., 2008). Na literatura é possível encontrar experimentos realizados no Brasil com animais saudáveis onde os níveis de frutosamina ficaram além do intervalo definido por Kaneko et al., (2008), por exemplo o de Soares et al., (2014) que trabalhando com ovelhas Dorper antes e após o parto em sistema semiextensivo em Pernambuco obtiveram médias de 143,79 a 191,3 $\mu \mathrm{mol} \mathrm{L}^{-1}$, e Santos et al., (2017) em experimento com ovelhas Santa Inês gestantes suplementadas com propilenoglicol em Minas Gerais encontraram média de $328,2 \mu \mathrm{mol} \mathrm{L}^{-1}$ para este metabólito.

Kaneko et al., (2008) não preconiza intervalos de referência para lipoproteínas, não havendo valores de comparação para os resultados obtidos neste estudo. Para o metabólito HDL (Lipoproteína de alta densidade) o intervalo de referência obtido foi de 10 a 76,7 $\mathrm{mg} \mathrm{dL}$ enquanto que para LDL (Lipoproteína de baixa densidade) o intervalo obtido foi de 1,2 a $87 \mathrm{mg}$ dL. Os níveis de HDL e LDL variam muito de acordo com o aporte energético da dieta, principalmente a concentração de ácidos graxos não esterificados. Comumente dietas com teores elevados de gordura resultam em maiores concentrações destes metabólitos (Varanis et al., 2018; Gressler et al., 2015). Santos et al., (2017) avaliando ovelhas Santa Inês gestantes suplementadas com propilenoglicol obteve média de $37,43 \mathrm{mg} \mathrm{dL}^{-1}$ para HDL e 19,84 $\mathrm{mg} \mathrm{dL}^{-1}$ para LDL. Gressler et al., (2015) trabalhando com ovelhas mestiças no Mato Grosso do Sul obtiveram valores entre 29,79 a $34,93 \mathrm{mg} \mathrm{dL}^{-1}$ para HDL e 18,5 a 22,29 $\mathrm{mg} \mathrm{dL}^{-1}$ para LDL. Borburema et al., (2012) utilizando borregos Santa Inês encontraram média de $64,8 \mathrm{mg} \mathrm{dL}^{-1}$ em seu experimento. Todos os resultados encontrados na literatura foram coerentes com o intervalo de referência determinado neste estudo.

Para VLDL (Lipoproteína de muito baixa densidade) o intervalo de referência foi de 1 a $16,4 \mathrm{mg} \mathrm{dL}^{-1}$. Nasciutti et al., (2012) trabalhando com ovelhas Santa 
Parâmetros de metabólitos bioquímicos em ovinos criados no Brasil

Inês nos períodos pré e pós parto encontraram médias entre 4,87 e 9,63 $\mathrm{mg} \mathrm{dL}^{-1}$ para este metabólito. Santos et al., (2015) em experimento com cordeiros Santa Inês avaliados do nascimento ao desmame obtiveram média de $6,94 \mathrm{mg} \mathrm{dL}^{-1}$, ambos os experimentos foram conduzidos em Uberlândia, Minas Gerais. A VLDL é responsável pelo transporte de triglicerídeos na corrente sanguínea, portanto é esperado que se comportem de maneira semelhante (Santos et al., 2015).

Para definir o perfil proteico foram obtidos dados de proteínas totais, ácido úrico, ureia, albumina e creatinina (Tabela 2).

Tabela 2 - Intervalos de referência para metabólitos proteicos de ovinos

\begin{tabular}{lcccc}
\hline Metabólito & $\mathbf{N}^{\mathbf{1}}$ & $\begin{array}{c}\text { Intervalos definidos } \\
\text { neste estudo }\end{array}$ & $\begin{array}{c}\text { Intervalos definidos por Kaneko } \\
\text { et al., (2008) }\end{array}$ & $\begin{array}{c}\text { Unidade } \\
\text { Proteínas totais }\end{array}$ \\
Acido úrico & 4235 & $3,1-10,7$ & $6-7,9$ & $\mathrm{mg} \mathrm{dL}^{-1}$ \\
Ureia & 4043 & $0-1,7$ & $0-1,9$ & $\mathrm{mg} \mathrm{dL}^{-1}$ \\
Albumina & 4134 & $10-92$ & $17-43$ & $\mathrm{~g} \mathrm{dL}^{-1}$ \\
Creatinina & 4359 & $1,1-5,2$ & $2,4-3,0$ & $\mathrm{mg} \mathrm{dL}^{-1}$ \\
\hline
\end{tabular}

Para os dados de proteínas totais, a amplitude do intervalo de referência foi de $7,6 \mathrm{~g} \mathrm{dL}^{-1}$, enquanto o de Kaneko et al (2018) é de $1,9 \mathrm{~g} \mathrm{dL}^{-1}$. A concentração de proteínas totais no sangue reflete o status nutricional proteico de maneira muito confiável. Em animais não lactantes uma diminuição dos níveis deste metabólito pode indicar deficiência proteica na dieta (Rodrigues et al., 2017). Já no pós-parto Silva et al., (2013) afirmam que há uma diminuição nos valores de proteínas totais nos primeiros 30 dias de lactação, devido à maior produção de leite nesta fase. Gressler et al., (2015) trabalhando com ovelhas Santa Inês adultas em Mato Grosso do Sul encontraram médias entre 6,62 a 7,01 $\mathrm{g} \mathrm{dL}^{-1}$. Brito et al., (2006) trabalhando com ovelhas Lacaune gestantes confinadas no Rio Gande do Sul, obteve média de 6,46 g $\mathrm{dL}^{-1}$. Lima et al., (2016) 7,06 $\mathrm{g} \mathrm{dL}^{-1}$. Cardoso et al., (2010) observaram média de $6,75 \mathrm{~g} \mathrm{dL}^{-1}$ em ovelhas Santa Inês no pós parto.

Para o metabólito ácido úrico, foi observada amplitude de $1,7 \mathrm{mg} \mathrm{dL}^{-1}, 0,2 \mathrm{mg} \mathrm{dL} \mathrm{m}^{-1}$ menor que o intervalo de Kaneko et al (2008). O ácido úrico é utilizado pelos microrganismos ruminais como fator de crescimento microbiano, sendo transformado em amônia e usado para sintetizar proteína microbiana (Paula, 2015). Variações na concentração sanguínea deste metabólito entre animais de idades diferentes podem ser atribuídas à diferenças na alimentação, e aumentam de acordo com a qualidade nutricional do alimento, indicando atividade ruminal recente (Neto et al., 2017). Na literatura encontram-se trabalhos como o de Sá et al., (2014), que trabalhando com borregos sem padrão racial definido no estado do Tocantins observaram concentração média de $0,26 \mathrm{mg}$ $\mathrm{dL}^{-1}$ para este metabólito enquanto Araújo et al., (2012) relataram, para carneiros adultos sem padrão racial definido, níveis entre 0,11 a $0,16 \mathrm{mg} \mathrm{dL}^{-1}$.
A amplitude do intervalo para ureia foi de $82 \mathrm{mg}$ $\mathrm{dL}^{-1}$, enquanto o preconizado por Kaneko et al., (2008) é de $29 \mathrm{mg} \mathrm{dL}^{-1}, 2,8$ vezes menor. Cerca de $70 \%$ da proteína ingerida é transformada em amônia no rumen, para ser utilizada como fonte de nitrogênio na síntese de aminoácidos e proteína microbiana, e, a fração da amônia não utilizada no rúmen é absorvida e direcionada ao fígado para ser transformada em ureia, que circula na corrente sanguínea. Sendo assim, a concentração de ureia plasmática tem relação direta com o aporte proteico e a proporção de proteína degradável no rumen contida na ração (Neto et al., 2017; Gressler, 2015).

Rabassa et al., (2009) obtiveram média de 30,68 $\mathrm{mg} \mathrm{dL} \mathrm{L}^{-1}$ para fêmeas Corriedale criadas a pasto no Rio Grande do Sul. Gressler et al., (2015) trabalhando com ovelhas mestiças Santa Inês relataram valores de 43,69 a $52,57 \mathrm{mg} \mathrm{dL}^{-1}$, enquanto Balaro, Cardoso e Peneiras (2012) em estudo conduzido no Rio de Janeiro com animais de aproximadamente 90 dias, encontraram o valor médio de $38,75 \mathrm{mg} \mathrm{dL} \mathrm{dL}^{-1}$. Dados encontrados na literatura de trabalhos conduzidos com ovelhas gestantes apresentam, em sua maioria, níveis de ureia acima do limite preconizado por Kaneko et al., (2008). Para ovelhas da raça Santa Inês Araújo (2009) observou valores entre 13,33 a 32,85 $\mathrm{mg} \mathrm{dL}^{-1}$, Cardoso et al., (2010) obtiveram nível médio de 40,48 $\mathrm{mg} \mathrm{dL}^{-1}$, Lima et al., (2016) média de 45,75 $\mathrm{mg} \mathrm{dL}^{-1}$ e Oliveira et al., (2014) média de 49,8 $\mathrm{mg} \mathrm{dL} \mathrm{d}^{-1}$. Ao final da gestação espera-se que as fêmeas diminuam a ingestão de alimentos, que pode levar ao aumento de proteólise endógena para uso como fonte energética, causando aumento nas concentrações de ureia (Feijó et al., 2014). Os níveis de ureia aumentados no fim da gestação tendem a diminuir com o avanço da fase de lactação, sendo possível associar essa diminuição ao balanço proteico negativo que o animal enfrenta nessa fase (Varanis, 2018; Silva et al., 2013) Cardoso et al., (2010) e Lima et al., (2016) avaliando ovelhas da raça Santa 
Inês observaram, aos 30 dias pós parto, valores médios de $26,48 \mathrm{mg} \mathrm{dL}^{-1}$ e $41,82 \mathrm{mg} \mathrm{dL}^{-1}$, respectivamente.

Para os níveis de albumina foi obtida amplitude de 4,1 $\mathrm{g} \mathrm{dL}^{-1}$, 6,8 vezes maior que o de Kaneko et al (2008). A albumina é a proteína mais abundante no plasma e seus níveis indicam, por meio de alterações lentas, o aporte proteico da dieta fornecida ao animal. Para que sejam observadas alterações significativas na sua concentração, é necessário um período de observação de pelo menos 30 dias (Varanis, 2018; Silva, 2019). Na literatura há trabalhos nacionais como o de Gouveia et al., (2015) que observaram o valor médio de $3,13 \mathrm{~g} \mathrm{dL}^{-1}$ para albumina em animais sem padrão racial definido com idade média de seis meses. Para ovinos Santa Inês Araújo (2009) observou valores entre 2,9 e 3,8 $\mathrm{g} \mathrm{dL}^{-1}$ para ovelhas prenhes e vazias criadas no estado de São Paulo, Borburema et al., (2012) obtiveram média de $3,02 \mathrm{~g} \mathrm{dL}^{-1}$ para borregos inteiros confinados e Marques (2007) obteve $2,78 \mathrm{~g} \mathrm{dL}^{-1}$ para borregos castrados.
O intervalo de creatinina teve amplitude de 1,3 $\mathrm{mg} \mathrm{dL} \mathrm{L}^{-1}$ enquanto o de Kaneko et al (2008) é de 0,7 mg $\mathrm{dL}^{-1}$. A creatinina é excretada exclusivamente por via renal, portanto sua concentração plasmática reflete a taxa de filtração glomerular. Altos níveis deste metabólito podem indicar deficiência na função renal (Varanis, 2018; Satake et al., 2018). Trabalhando com borregos Santa Inês, Marques (2007) obteve média de $1,86 \mathrm{mg} \mathrm{dL}^{-1}$ para este metabólito. Araújo (2009) relatou valores variando entre 0,9 a $1,3 \mathrm{mg} \mathrm{dL}^{-1}$ para ovelhas Santa Inês vazias e gestantes enquanto Santos et al., (2014) observaram avaliando ovelhas Morada Nova, média de $0,87 \mathrm{mg} \mathrm{dL}^{-1}$ aos 15 dias de lactação.

Para definir o perfil metabólico enzimático foram coletados dados de aspartato aminotransferase (AST), gama glutamil transferase (GGT), e fosfatase alcalina (ALP) (Tabela 3). As enzimas AST, GGT e ALP são analisadas para determinar função hepática.

Tabela 4 - Intervalos de referência para metabólitos enzimáticos de ovinos.

\begin{tabular}{ccccc}
\hline Metabólito & $\mathrm{N}^{1}$ & $\begin{array}{c}\text { Intervalos definidos } \\
\text { neste estudo }\end{array}$ & $\begin{array}{c}\text { Intervalos definidos por } \\
\text { Kaneko } \text { et al., (2008) }\end{array}$ & Unidade \\
\hline AST & 3513 & $41-298$ & $60-280$ & $\mathrm{U} \mathrm{L}^{-1}$ \\
GGT & 3523 & $25-146$ & $20-52$ & $\mathrm{U} \mathrm{L}^{-1}$ \\
ALP & 3167 & $49-826,9$ & $68-387$ & $\mathrm{U} \mathrm{L}^{-1}$ \\
\hline
\end{tabular}

$\mathrm{N}^{1}$ - número amostral. AST: aspartato aminotransferase; GGT: gama glutamiltransferase; ALP: fosfatase alcalina.

A partir dos dados de AST, a amplitude do intervalo obtido foi de $257 \mathrm{U} \mathrm{L}^{-1}$ enquanto o de Kaneko et al (2008) teve amplitude de $220 \mathrm{U} \mathrm{L}^{-1}$. A aspartato aminotransferase (AST) apresenta correlação positiva com as atividades da glândula mamária, produção de leite, problemas hepáticos e cardíacos, sendo dosada na avaliação principalmente de doenças hepáticas. Sua concentração pode ser elevada nos primeiros dias de vida devido ao maior consumo de colostro rico em gorduras (Feijó et al.,; 2014; Santos et al., 2015; Varanis, 2018). Para ovinos com idade média de seis meses, sem padrão racial definido, Gouveia et al., (2015) observaram valor médio de 106,34 U L-1. Trabalhando com fêmeas mestiças Corriedale x Texel, Rabassa et al., (2009) observou média de 73,2 U L-1 . Para animais da raça Morada Nova aos 15 dias de lactação, Santos et al., (2014) observaram média de $101,45 \mathrm{U} \mathrm{L}^{-1}$.

A amplitude do intervalo de GGT foi de $121 \mathrm{U}$ $\mathrm{L}^{-1}$ enquanto o de Kaneko et al (2008) é de $32 \mathrm{U} \mathrm{L}^{-1}$, 3,7 vezes menor. $\mathrm{O}$ aumento nos níveis de GGT podem indicar, assim como a AST, desordens hepáticas. Uma mobilização intensa de reservas lipídicas também pode causar aumento nos níveis de GGT, fazendo com que sejam um bom critério de avaliação para desordens no pós-parto, como esteatose hepática e toxemia (Araújo et al., 2012). Para cordeiros da raça Santa Inês com idade média de 100 dias, Borburema et al., (2012) observaram o valor de 51,79 U L-1 . Araújo (2009) observou média de 56,39 U L-1 para ovelhas Santa Inês no início do terço final de gestação, enquanto Nascimento (2015) relataram valores médios de 54,53 e $57,4 \mathrm{U} \mathrm{L}^{-1}$ para ovelhas aos 30 dias de lactação.

Finalmente, para fosfatase alcalina, a amplitude obtida no intervalo foi de 777,9 $\mathrm{U} \mathrm{L}^{-1}$, enquanto a de Kaneko et al., (2008) é de $319 \mathrm{U} \mathrm{L}^{-1}, 2,4$ vezes menor. Assim como as enzimas anteriores, a fosfatase alcalina também reflete o funcionamento hepático, portanto valores altos podem indicar a ocorrência de hepatopatias. Seus níveis séricos podem sofrer alterações e se apresentar elevados em situações que envolvam reabsorção óssea (Araújo et al., 2012). Santos et al., (2015) trabalhando com cordeiros Santa Inês entre 9 e 90 dias de idade observaram média de 449, $95 \mathrm{U} \mathrm{L}^{-1}$. Trabalhando com ovelhas Morada Nova, no período de 60 a 7 dias antes do parto, Santos et al., (2014) observaram média de 109,13 U L-1. Nasciutti et al. (2012) relatou a concentração média de $66,67 \mathrm{U} \mathrm{L}^{-1}$, 28 dias após o parto em ovelhas Santa Inês.

De modo geral, os resultados obtidos neste estudo foram mais amplos que os valores preconizados por Kaneko et al., (2008), com destaque para o metabólito frutosamina que teve um intervalo 83 vezes mais amplo. A concentração sanguínea dos metabólitos sofre alterações por influencia de vários fatores intrínsecos 
ao animal e ao ambiente, principalmente da dieta. A adaptação do organismo animal a uma determinada configuração ambiental influencia no funcionamento das vias metabólicas, podendo ocasionar variações nos níveis considerados normais, entretanto não necessariamente essas variações são sinônimos de doenças ou desordens metabólicas, devendo ser analisadas levando em consideração o ambiente no qual o animal se encontra (Neto et al., 2017; Varanis, 2018).

Os dados encontrados na literatura nacional corroboram a hipótese de que os valores definidos por Kaneko et al (2008) não refletem apropriadamente a realidade dos ovinos brasileiros, visto que a maior parte dos dados apresentados encontrou-se fora dos intervalos preconizados pelo mesmo e dentro dos intervalos defini- dos neste estudo. Levando em consideração apenas tais valores, isso poderia conduzir o pesquisador a equívocos na interpretação dos resultados, entretanto, após avaliação cuidadosa, os animais foram considerados saudáveis mesmo diferindo do intervalo referêncial utilizado, evidenciando assim a necessidade de uma tabela nacional, que proporcione maior acurácia na interpretação de dados de perfis metabólicos.

\section{Conclusão}

Foram definidos intervalos de referência de valores de metabólitos energéticos, proteicos, minerais e enzimáticos para ovinos, sendo estes mais amplos que os valores internacionais comumente utilizados, abrangendo melhor resultados obtidos exclusivamente no Brasil.

\section{Referências}

Alvarenga, A. B. B. 2011. Resposta fisiológica de diferentes genótipos de carneiros relacionada a processos adaptativos ambientais em clima tropical úmido durante o outono e inverno. Brasília: Universidade de Brasília, 89f. il. Tese Doutorado. Disponível em: https://repositorio. unb.br/handle/10482/10993.

Angeli, N. C. 2014. Metabolismo de lipídeos em ruminantes. Seminário apresentado na disciplina Bioquímica do Tecido Animal, Programa de Pós-Graduação em Ciências Veterinárias, Universidade Federal do Rio Grande do Sul. 6 p. Disponível em: https://www.ufrgs.br/lacvet/site/ wp-content/uploads/2014/08/lipideos_ruminantes.pdf.

Araújo, C. A. S. C. 2009. Estudo comparativo do perfil metabólico e hormonal de ovelhas com gestação única, gemelar e não gestantes alimentadas com dieta de alta densidade energética. São Paulo: Universidade de São Paulo. 212f. Dissertação Mestrado. Doi: https:// dx.doi.org/10.11606/D.10.2010.tde-21092010-162531.

Araújo, P. B.; Andrade, R. D. P. X.; Ferreira, M. A.; Batista, A. M. V.; Caravalho, C. C. D.; Soares, P. C. 2012. Efeito da substituição do feno de capim tifton (Cynodon spp.) por casca de mamona (Ricinus communis) em dietas a base de palma forrageira (Nopalea cochenilifera Salm dick) sobre o metabolismo energético, proteico e mineral em ovinos. Brazilian Journal of Veterinary Medicine, 34(4), 327-335. Disponível em: http:// rbmv.org/index.php/BJVM/article/view/738.

Balaro, M. F. A.; Cardoso, E. C.; Peneiras, A. B. V. 2012. Ganho de peso e perfil metabólico sanguíneo de cordeiros alimentados com dietas contendo gordura protegida. Agroecossistemas, 4(1): 42-49. Doi: https://doi.org/10.18542/ragros.v4i1.1049.

Borburema, J. B.; Cezar, M. F.; Marques, D. D.; Cunha, M. G. G.; Pereira Filho, J. M.; Sousa, W. H.; Furtado, D. A.; Costa, R. G. 2012. Efeito do regime alimentar sobre o perfil metabólico de ovinos Santa Inês em confinamento. Arquivo Brasileiro de Medicina Veterinária e Zootecnia, 64(4): 983-990. Doi: https://dx.doi.org/10.1590/S010209352012000400027.

Brito, M. A.; González, F. H. D.; Ribeiro, L. A. O.; Campos, R.; Lacerda, L. D. A.; Barbosa, P. R.; Bergmann, G. P. 2006. Composição do sangue e do leite em ovinos leiteiros do sul do Brasil: variações na gestação e na lactação. Ciência rural, 36(3): 942-948. Doi: http://dx.doi.org/10.1590/ S0103-84782006000300033.
Cardoso, E. C.; Oliveira, D. R.; Dourado, A. P.; Araújo, C. V.; Ortolani, E. L.; Brandão, F. Z. 2010. Peso e condição corporal, contagem de OPG e perfil metabólico sanguíneo de ovelhas da raça Santa Inês no periparto, criadas na região da Baixada Litorânea do Estado do Rio de Janeiro. Revista Brasileira de Ciência Veterinária, 17(2): 77-82. Doi: http://dx.doi.org/10.4322/rbcv.2014.148.

Feijó, J. O.; Pezzaroli, D.; Silva, L. G. C.; Aragão, R. B.; Martins. C. F; Pereira, R. A.; Ferreira, M. B.; Pino, F. A. B. D.; Rabassa, V. R.; Corrêa, M. N. 2014. Avaliação de parâmetros bioquímicos clínicos de ovelhas do grupo genético pantaneiro gestantes e não gestantes. Brazilian Journal of Veterinary Research and Animal Science, 51(2): 111-117. Doi: http://dx.doi.org/10.11606/issn.1678-4456.v51i2p111-117.

Friedewald, W. T.; Levv, R. I.; Fredrickson, D. S. 1972. Estimation of the concentration of low-density lipoprotein in plasma, without use of the preparative ultracentrifuge. Clinical Chemistry. 18, 499-502. Disponível em: http://clinchem.aaccjnls.org/content/clinchem/18/6/499.full.pdf.

Gouveia, L. N. F.; Maciel, M. V.; Soares, P. C.; Silva Neto, I. F.; Gonçalves, D. N. A.; Batista, A. M. V.; Carvalho, F. F. R. 2015. Perfil metabólico de ovinos em crescimento alimentados com dietas constituídas de feno ou silagem de maniçoba e palma forrageira. Pesquisa Veterinária Brasileira, 35(1): 5-9. Doi: http://dx.doi.org/10.1590/S0100736X2015001300002.

Gressler, M. A. L.; Souza, M. I. L.; Souza, A. S.; Filiú, W. F. O.; Aguena, S. M.; Franco, G. L. 2015. Respostas bioquímicas de ovelhas submetidas à flushing de curto prazo em região subtropical. Revista Brasileira de Saúde e Produção Animal, 16(1): 210-222. Doi: https://doi.org/10.1590/ S1519-99402015000100022.

Homem Junior, A. C.; Ezequiel. J. M. B; Galati, R. L.; Gonçalves, J. D. S.; Santos, V. C.; Sato, R. A. 2010. Grãos de girassol ou gordura protegida em dietas com alto concentrado e ganho compensatório de cordeiros em confinamento. Revista Brasileira de Zootecnia, 39(3): 563-571. Doi: http://dx.doi.org/10.1590/S1516-35982010000300016.

Kaneko, J. J.; Harvey, J. W.; Bruss, M. L. 2008. Clinical Biochemistry of Domestic Animals. 6.ed. Academic Press, San Diego, CA, EUA.

Kozloski, G. V. 2017. Bioquímica dos ruminantes. 3.ed. Fundação de Apoio a Tecnologia e Ciência - Editora UFSM, RS, BR. 
Silva, D. A. P. et al.

Libardi, K. D. C.; Costa, P. B.; Oliveira, A. A. M. A.; Cavilhão, C.; Hermes, P. R.; Ramella, J. R. P. 2018. Perfil metabólico de cordeiros Santa Inês terminados em confinamento com manejo alimentar restritivo e ad libitum. Ciência Animal Brasileira, 19: 1-15. Doi: http://dx.doi. org/10.1590/1809-6891v19e-31227.

Lima, E. H. F.; Mendonça, C. L.; Cajueiro, J. F. P.; Carvalho, C. C. D.; Soares, P. C.; Souto, R. J. C.; Drummond, A. R. F.; Afonso, J. A. B. 2016. Efeito da monensina sódica sobre o perfil metabólico de ovelhas antes e após o parto. Ciência Animal Brasileira, 17(1): 105-118. Doi: http:// dx.doi.org/10.1590/1089-6891v17i128370.

Marques, K. S. 2007. Perfil metabólico de cordeiros em pastejo submetidos a diferentes ambientes e suplementações alimentares no semi-árido paraibano. Patos: Universidade Federal de Campina Grande. 44f. Dissertação Mestrado. Disponível em: http://dspace.sti.ufcg.edu. br:8080/jspui/handle/riufcg/9124.

Nascimento, P. M.; Morgado, A. A.; Nunes, G. R.; Nikolaus, J. P.; Weigel, R. A.; Lima, A. S.; Sucupira, M. C. A. 2015. Metabolismo oxidativo e perfil bioquímico de ovelhas santa inês no período periparto: efeito da suplementação parenteral com vitamina E. Semina: Ciências Agrárias, 36(3): 1397-1407. Doi: http://dx.doi.org/10.5433/16790359.2015v36n3p1397.

Nasciutti, N.; Tsuruta, S.; Oliveira, R.; Bisinoto, M.; Headley, S.; Mundim, A.; Noleto, P.; Saut, J. P. 2012. Perfil metabólico em ovelhas Santa Inês, com baixo escore de condição corporal, no periparto. Boletim De Indústria Animal, 69(2): 137-145. Disponível em: http://www.iz.sp. gov.br/bia/index.php/bia/article/view/1032.

Neto, J. G.; Pedreira, M. S.; Silva, H. G. O.; Alves, E. M.; Santos, E. D. J.; Silva, Á. C.; Corrêa, Y. R. 2017. Tipos de ureia e fontes de carboidratos nas dietas de cordeiros: síntese de proteína microbiana e balanço de nitrogênio. Revista Electrónica de Veterinaria, 18(9): 1-15. Disponível em: https://www.redalyc.org/articulo.oa?id=63653009064.

Oliveira, R. P. M.; Maduro, A. H. P.; de Oliveira, F. F.; Lima, E. S. 2014. Perfil metabólico de ovelhas Santa Inês em diferentes fases de gestação criadas em sistema semi-intensivo no Estado do Amazonas. Ciência Animal Brasileira, 15(1): 81-86. Doi: http://dx.doi.org/10.5216/ cab.v15i1.15720.

Paula, C. G. 2015. Suplementação com melaço de soja na dieta de ovinos: parâmetros sanguíneos, consumo, digestibilidade e comportamento ingestivo. Uberlândia: Universidade Federal de Uberlândia. 61f. Dissertação Mestrado. Disponível em: http://repositorio.ufu.br/ handle/123456789/13154.

Payne, J. M.; Dew, S. M.; Manston, R.; Faulks. M. 1970. The use of the metabolic profiles test in dairy herds. Veterinary Records, 87: 150-158. Doi: http://dx.doi.org/10.1136/vr.87.6.150.

Rabassa, V. R.; Tabeleão, V. C.; Scheneider, A.; Menezes, L. M.; Schossler, E.; Severo, N.; Corrêa, M. N. 2009. Avaliação metabólica de ovelhas de cria mantidas em campo nativo durante o período de outono/inverno. Current Agricultural Science and Technology, 15: 1-4. Doi: http:// dx.doi.org/10.18539/cast.v15i1-4.1999.

Ribeiro, L. A. B., González, F. H. D.; Conceição, T. R.; Brito, M. A.; LA Rosa, V. L.; Campos, R. 2003. Perfil metabólico de borregas Corriedale em pastagem nativa do Rio Grande do Sul. Acta Scientiae Veterinariae, 31(3): 167-170. Doi: http://dx.doi.org/10.22456/1679-9216.17161.
Rodrigues, O. G.; Marques, K. B.; de Sousa, B. B. 2017. Perfil mineral sérico de cordeiros submetidos a diferentes suplementações alimentares e a diferentes tipos de ambientes em pastejo no semiárido. Agropecuária Científica no Semiárido, 12(4): 332-338. Doi: http:// dx.doi.org/10.30969/acsa.v12i4.701.

Sá, H. M.; Teles, T. L.; Borges, I.; Macedo Jr, G. L.; Silva, S. P. 2014. Perfil metabólico em ovinos alimentados com inclusões crescentes da torta do babaçu na dieta. Veterinária Notícias, 20(2), 48-56. Doi: https:// doi.org/10.14393/VTv20n2a2014.26804.

Santos, F. M. S. C.; Soares, P. C.; Mesquita, E. P.; Oliveira Filho, E. F.; Guido, S. I.; Alves, K. H. G.; Bartolomeu, C. C.; Amorim, M. J. A. A. L. 2014. Perfil bioquímico em ovelhas da raça Morada Nova nos períodos de gestação, parto e pós parto. Ciência Veterinária nos Trópicos. 17(1): 24-29. Disponível em: https://www.bvs-vet.org.br/vetindex/periodicos/ ciencia-veterinaria-nos-tropicos/17-(2014)-1-2/perfil-bioquimico-emovelhas-da-raca-morada-novanos-periodos-de-gestac/.

Santos, R. P.; Souza, L. F; Souza, J. T. L.; Andrade, M. E. B.; Macedo Jr, G. L.; Silva, S. P. 2015. Parâmetros sanguíneos de cordeiros em crescimento filhos de ovelhas suplementadas com níveis crescentes de propilenoglicol. Revista Brasileira de Ciências Agrárias, 10(3): 473-478. Doi: http://dx.doi.org/10.5039/agraria.v10i3a4924.

Santos, R. P.; Macedo Jr, G. L.; Silva, S. P.; Souza, L. F.; Andrade, M. E. B. 2017. A suplementação com propilenoglicol melhora o metabolismo energético em ovelhas gestantes. Revista Brasileira de Ciências Agrárias. 12(4): 561-566. Doi: http://dx.doi.org/10.5039/agraria.v12i4a5484.

Satake, F.; Malta, K. C.; Silva, S. L. 2018. Avaliação da transferência de imunidade passiva e de constituintes séricos de cordeiros Santa Inês nascidos. Pesquisa Veterinária Brasileira, 38(2): 294-299. Doi: http:// dx.doi.org/10.1590/1678-5150-pvb-3968.

Silva, J. S. C.; Guaraná, E. L. S.; Lemos, V. F.; Soares, P. C.; Afonso, J. A. B.; Mendonça, C. L. 2013. Metabolismo energético, proteico e mineral de ovelhas Santa Inês hígidas e com mastite subclínica. Pesquisa Veterinária Brasileira, 33(9): 1087-1096. Doi: https://doi.org/10.1590/ S0100-736X2013000900007.

Silva, M. S. C.; Rodrigues, D. S.; Tuerlinckx, S.; Siqueira, C. M. G. 2019. Perfil bioquímico de albumina e colesterol em vacas de invernar submetidas a suplementação. Anais do Salão Internacional de Ensino, Pesquisa e Extensão, Universidade Federal do Pampa, Santana do Livramento, RS, Brasil, 10. Disponível em: http://200.132.146.161/ index.php/siepe/article/view/38760.

Soares, F. A. P; Neto, A. V. B.; Freitas, I. B.; Carvalho, C. C. D., Barbosa, J. D.; Soares, P. C. 2014. Perfil sérico de alguns constituintes sanguíneos de ovelhas da raça Dorper no período gestacional e pós-parto. Revista de Ciências Agrárias Amazonian Journal of Agricultural and Environmental Sciences, 57(3): 266-272. Disponível em: https://periodicos.ufra.edu. br/index.php/ajaes/article/view/921.

Solberg, H. E. 2006. RefVal 4.11 software user's guide. RefVal, Rykkinn, Norway.

Varanis, L. F. M. 2018. Prospecção de metabólitos sanguíneos referenciais para ovinos em distintas categorias. Uberlândia: Universidade Federal de Uberlândia. 88 f. Dissertação Mestrado. Doi: http://dx.doi. org/10.14393/ufu.di.2018.787. 